\title{
Research Article Synchronization of Coupled Networks with Mixed Delays by Intermittent Control
}

\author{
Kun Yuan, ${ }^{1}$ Jinde Cao, ${ }^{2}$ and Shumin Fei ${ }^{1}$ \\ ${ }^{1}$ School of Automation, Southeast University, Nanjing 210096, China \\ ${ }^{2}$ Department of Mathematics, Southeast University, Nanjing 210096, China \\ Correspondence should be addressed to Kun Yuan, kyuan@seu.edu.cn
}

Received 3 May 2012; Revised 26 June 2012; Accepted 26 June 2012

Academic Editor: Zhiwei Gao

Copyright (C) 2012 Kun Yuan et al. This is an open access article distributed under the Creative Commons Attribution License, which permits unrestricted use, distribution, and reproduction in any medium, provided the original work is properly cited.

The synchronization of coupled networks with mixed delays is investigated by employing Lyapunov functional method and intermittent control. A sufficient condition is derived to ensure the global synchronization of coupled networks, which is controlled by the designed intermittent controller. Finally, a numerical simulation is constructed to justify the theoretical analysis.

\section{Introduction}

Various large-scale and complicated systems can be modelled by complex networks, such as the Internet, genetic networks, ecosystems, electrical power grids, and the social networks. A complex network is a large set of interconnected nodes, which can be described by the graph with the nodes representing individuals in the graph and the edges representing the connections among them. The most remarkable recent advances in study of complex networks are the developments of the small-world network model [1] and scale-free network model [2], which have been shown to be very closer to most real-world networks as compared with the random-graph model [3, 4]. Thereafter, small-world and scale-free networks have been extensively investigated.

The dynamical behaviors of complex networks have become a focal topic of great interest, particularly the synchronization phenomena, which is observed in natural, social, physical, and biological systems and has been widely applied in a variety of fields, such as secure communication, image processing, and harmonic oscillation generation. It is noted that the dynamical behavior of a complex network is determined not only by the dynamical 
rules governing the isolated nodes, referred to as self-dynamics, but also by information flow along the edges, which depends on the topology of the network. Synchronization in an array of linearly coupled dynamical systems was investigated in [5]. Later, many results on local, global, and partial synchronization in various coupled systems have also been obtained in [6-15]. As a special case of coupled systems, coupled neural networks with time delay have also been found to exhibit complex behaviors. The estimation and diagnosis for time delay systems are discussed in $[16,17]$, and synchronization for coupled neural networks with time delay has been investigated by many researchers, for example, [8-15].

In the case that the whole network cannot synchronize by itself, some controllers should be designed and applied to force the network to synchronize. Recently, another interesting intermittent control was introduced and studied, that is, the control time is periodic, and in any period the time is composed of work time and rest time. It is a straightforward engineering approach to process control of any typelan approach that has been used for a variety of purposes in such engineering fields as manufacturing, transportation, and communication. Intermittent control has been introduced to control nonlinear dynamical systems [18] and has been studied in [19-26]. In [18], the authors investigated numerically chaos synchronization under the condition that the interacting systems, that is, master and slave systems are coupled intermittently. In [19, 20], the stabilization problems of chaotic systems with or without delays by periodically intermittent control were discussed. Huang et al. discussed the synchronization of coupled chaotic systems with delay by using intermittent state feedback in [21]. In [25], the authors synchronize coupled networks using pinning control and intermittent control. In [26], cluster synchronization was studied for coupled networks without time delay using adaptive intermittent control.

Another type of time delays, namely, distributed delays, has begun to receive research attention. The main reason is that a neural network usually has a spatial nature due to the presence of an amount of parallel pathways of a variety of axon sizes and lengths, and it is desirable to model them by introducing continuously distributed delays over a certain duration of time, such that the distant past has less influence compared to the recent behavior of the state [27]. Therefore, both discrete and distributed time delays should be taken into account [28-33]. Although synchronization has been investigated under intermittent control, $[25,26]$, there is still no theoretical result of synchronization for coupled networks with mixed delay.

Motivated by the above discussion, the intermittent controller will be designed to achieve the synchronization for coupled networks with mixed delay. The rest of the paper is organized as follows. In Section 2, some preliminary definitions and lemmas are briefly outlined. Some synchronization criteria are given and intermittent controller are designed in Section 3. An illustrative simulation is given to verify the theoretical analysis in Section 4. Conclusions are finally drawn.

\section{Notations}

$\mathbb{R}^{n}$ is the $n$-dimensional Euclidean space; $\mathbb{R}^{m \times n}$ denotes the set of $m \times n$ real matrix. $I$ is the identity matrix with appropriate dimension, and the superscript " $T$ " represents the transpose. Matrix dimensions, if not explicitly stated, are assumed to be compatible for algebraic operations. 


\section{Model Description and Preliminaries}

Consider a dynamical network consisting of $N$ identical and diffusively coupled nodes, with each node being an $n$-dimensional delayed neural network. The state equations of the network are

$$
\begin{aligned}
\dot{x}_{i}(t)= & -D x_{i}(t)+A f\left(x_{i}(t)\right)+B g\left(x_{i}(t-\tau)\right)+C \int_{t-\tau}^{t} h\left(x_{i}(v)\right) d v \\
& +I(t)+\sum_{j=1, j \neq i}^{N} G_{i j} \Gamma\left(x_{j}(t)-x_{i}(t)\right),
\end{aligned}
$$

where $x_{i}(t)=\left(x_{i 1}(t), x_{i 2}(t), \ldots, x_{i n}(t)\right)^{T} \in \mathbb{R}^{n}$ is the state vector of the $i$ th node; $D=$ $\operatorname{diag}\left(d_{1}, d_{2}, \ldots, d_{n}\right)>0$ denotes the rate with which the cell $i$ resets its potential to the resting state when isolated from other cells and inputs; $A \in \mathbb{R}^{n \times n}, B \in \mathbb{R}^{n \times n}$, and $C \in \mathbb{R}^{n \times n}$ represent the connection weight matrix, the discretely delayed connection weight matrix, and the distributively delayed connection weights, respectively; $f\left(x_{i}(\cdot)\right)=$ $\left[f_{1}\left(x_{i 1}(\cdot)\right), f_{2}\left(x_{i 2}(\cdot)\right), \ldots, f_{n}\left(x_{i n}(\cdot)\right)\right]^{T} \in \mathbb{R}^{n}, g\left(x_{i}(\cdot)\right)=\left[g_{1}\left(x_{i 1}(\cdot)\right), g_{2}\left(x_{i 2}(\cdot)\right), \ldots, g_{n}\left(x_{i n}(\cdot)\right)\right]^{T} \in$ $\mathbb{R}^{n}$ and $h\left(x_{i}(\cdot)\right)=\left[h_{1}\left(x_{i 1}(\cdot)\right), h_{2}\left(x_{i 2}(\cdot)\right), \ldots, h_{n}\left(x_{i n}(\cdot)\right)\right]^{T} \in \mathbb{R}^{n}$ are activation functions; $I(t)$ is the input vector of each node; $\Gamma \in \mathbb{R}^{n \times n}$ is the inner coupling matrix; $G=\left(G_{i j}\right)_{N \times N}$ is the coupling configuration matrix representing the topological structure of the network, where $G_{i j}$ is defined as follows: if there exists a connection between node $i$ and node $j, G_{i j}>0$, otherwise $G_{i j}=0(j \neq i)$, and the diagonal elements of matrix $G$ are defined by

$$
G_{i i}=-\sum_{j=1, j \neq i}^{N} G_{i j},
$$

which ensures the diffusion that $\sum_{j=1}^{N} G_{i j}=0$. Equivalently, network (2.1) can be rewritten in a form as follows:

$$
\begin{aligned}
\dot{x}_{i}(t)= & -D x_{i}(t)+A f\left(x_{i}(t)\right)+B g\left(x_{i}(t-\tau)\right)+C \int_{t-\tau}^{t} h\left(x_{i}(v)\right) d v \\
& +I(t)+\sum_{j=1}^{N} G_{i j} \Gamma x_{j}(t), \quad i=1,2, \ldots, N .
\end{aligned}
$$

Suppose that the coupled network (2.3) is connected in the sense that there are no isolated clusters, then the coupling matrix $G$ is irreducible.

Note that a solution to an isolated node satisfies

$$
\frac{d s(t)}{d t}=-D s(t)+A f(s(t))+B g(s(t-\tau))+C \int_{t-\tau}^{t} h(s(v)) d v+I(t) .
$$


To realize the synchronization of network (2.3), the intermittent strategy is selected, and the controlled network can be described by

$$
\begin{aligned}
\dot{x}_{i}(t)= & -D x_{i}(t)+A f\left(x_{i}(t)\right)+B g\left(x_{i}(t-\tau)\right)+C \int_{t-\tau}^{t} h\left(x_{i}(v)\right) d v \\
& +I(t)+\sum_{j=1}^{N} G_{i j} \Gamma x_{j}(t)+u_{i}, \quad i=1,2, \ldots, N
\end{aligned}
$$

where

$$
u_{i}=-k_{i}(t)\left(x_{i}(t)-s(t)\right)
$$

$k(t)$ is the intermittent linear state feedback control gain defined as follows:

$$
k_{i}(t)= \begin{cases}k_{i}, & n \omega \leq t \leq n \omega+\delta \\ 0, & n \omega+\delta<t \leq(n+1) \omega\end{cases}
$$

where $k_{i} \in R$ is a constant control gain, $\omega>0$ is the control period, and $\delta>0$ is called the control width. In this paper, our goal is to design suitable $\delta, \omega$, and $k_{i}$ such that network (2.5) synchronize with respect to the isolated node $s(t)$. Denote $e_{i}(t)=x_{i}(t)-s(t)$, then the following error dynamical system is obtained:

$$
\begin{aligned}
\dot{e}_{i}(t)= & -D e_{i}(t)+A\left[f\left(x_{i}(t)\right)-f(s(t))\right]+B\left[g\left(x_{i}(t-\tau)\right)-g(s(t-\tau))\right] \\
& +C \int_{t-\tau}^{t}\left[h\left(x_{i}(v)\right)-h(s(v))\right] d v+\sum_{j=1}^{N} G_{i j} \Gamma e_{j}(t)-k_{i} e_{i}(t), \quad n \omega \leq t \leq n \omega+\delta, \\
\dot{e}_{i}(t)= & -D e_{i}(t)+A\left[f\left(x_{i}(t)\right)-f(s(t))\right]+B\left[g\left(x_{i}(t-\tau)\right)-g(s(t-\tau))\right] \\
& +C \int_{t-\tau}^{t}\left[h\left(x_{i}(v)\right)-s(v)\right] d v+\sum_{j=1}^{N} G_{i j} \Gamma e_{j}(t), \quad n \omega+\delta<t \leq(n+1) \omega .
\end{aligned}
$$

(H) We assume that $f, g$, and $h$ are Lipschitz continuous functions; there exist positive constants $L_{f}, L_{g}$ and $L_{h}$ such that, for all $x, y \in \mathbb{R}^{m}$,

$$
\begin{aligned}
\|f(x)-f(y)\| & \leq L_{f}\|x-y\|, \\
\|g(x)-g(y)\| & \leq L_{g}\|x-y\|, \\
\|h(x)-h(y)\| & \leq L_{h}\|x-y\| .
\end{aligned}
$$


Definition 2.1. For any positive integers $p, q, r, s$, we define the Kronecker product of two matrices $A \in \mathbb{R}^{p \times q}, B \in \mathbb{R}^{r \times s}$ as follows:

$$
A \otimes B=\left[\begin{array}{ccc}
a_{11} B & \cdots & a_{1 q} B \\
\vdots & \ddots & \vdots \\
a_{p 1} B & \cdots & a_{p q} B
\end{array}\right] \in \mathbb{R}^{p r \times q s}
$$

Lemma 2.2. By the definition of Kronecker product, the following properties hold:

(1) $(A \otimes B)^{T}=A^{T} \otimes B^{T}$;

(2) $(\alpha A) \otimes B=A \otimes(\alpha B)$, where $\alpha$ is a real number;

(3) $(A \otimes B)(C \otimes D)=(A C) \otimes(B D)$.

Lemma 2.3. For any vectors $x, y \in \mathbb{R}^{m}$, and positive-definite matrix $Q \in \mathbb{R}^{m \times m}$, the following matrix inequality holds:

$$
2 x^{T} y \leq x^{T} Q x+y^{T} Q^{-1} y
$$

Lemma 2.4 (Jensen's inequality [34]). For any constant matrix $V \in \mathbb{R}^{m \times m}, V>0$, scalar $0<r(t)<r$, vector function $v:[0, r] \rightarrow \mathbb{R}^{m}$ such that the integrations concerned are well defined, then

$$
r(t) \int_{0}^{r(t)} \mathcal{v}^{T}(s) V \mathcal{v}(s) d s \geq\left(\int_{0}^{r(t)} \mathcal{v}(s) d s\right)^{T} V\left(\int_{0}^{r(t)} v(s) d s\right)
$$

Lemma 2.5 (Halanay inequality [35]). Let $V:[\mu-\tau, \infty) \rightarrow[0, \infty)$ be a continuous function such that

$$
\frac{d V(t)}{d t} \leq-a V(t)+b \max V_{t}
$$

is satisfied for $t \geq \mu$. If $a>b>0$, then

$$
V(t) \leq\left[\max V_{\mu}\right] \exp \{-r(t-\mu)\}, \quad t \geq \mu,
$$

where $\max V_{t}=\sup _{t-\tau \leq \theta \leq t} V(\theta)$, and $r>0$ is the smallest real root of the following equation:

$$
-r=-a+b \exp \{r \tau\}
$$

Lemma 2.6 (see [19]). Let $V:[\mu-\tau, \infty) \rightarrow[0, \infty)$ be a continuous function, such that

$$
\frac{d V(t)}{d t} \leq a V(t)+b \max V_{t}
$$


is satisfied for $t \geq \mu$. If $a>0, b>0$, then

$$
V(t) \leq \max V_{t} \leq\left[\max V_{\mu}\right] \exp \{(a+b)(t-\mu)\}, \quad t \geq \mu,
$$

where $\max V_{t}=\sup _{t-\tau \leq \theta \leq t} V(\theta)$.

\section{Criteria for Synchronization}

Theorem 3.1. Suppose that assumption $(H)$ holds. The controlled coupled network (2.5) globally synchronizes to (2.4) if there are positive definite matrix $P$, positive constants $\alpha, \beta, \gamma, a_{1}, a_{2}, b_{1}, b_{2}$ such that the following conditions hold:

(a) $I_{N} \otimes\left(Q+a_{1} P\right)+G \otimes \Gamma-K \otimes I_{n} \leq 0$,

(b) $I_{N} \otimes\left(Q-a_{2} P\right)+G \otimes \Gamma \leq 0$,

(c) $\beta^{-1} L_{g}^{2} I_{n}-b_{1} P \leq 0$,

(d) $\gamma^{-1} L_{h}^{2} I_{n}-b_{2} P \leq 0$,

(e) $a_{1}>b=b_{1}+\tau^{2} b_{2}$

(f) $\rho=r(\delta-\tau)-\left(a_{2}+b\right)(\omega-\delta)>0$,

where $Q=-P D+(\alpha / 4) P A A^{T} P+\alpha^{-1} L_{f}^{2} I_{n}+(\beta / 4) P B B^{T} P+(\gamma / 4) P C C^{T} P+a_{1} P, K=$ $\operatorname{diag}\left(k_{1}, k_{2}, \ldots, k_{N}\right)$ and $r$ is the positive solution of $-r=-a_{1}+b e^{r \tau}$.

Proof. Consider the following Lyapunov function:

$$
V(t)=\frac{1}{2} \sum_{i=1}^{N} e_{i}(t)^{T} P e_{i}(t)=\frac{1}{2} e^{T}\left(I_{N} \otimes P\right) e(t)
$$

where $e(t)=\left[e_{1}^{T}(t), e_{2}^{T}(t), \ldots, e_{N}^{T}(t)\right]^{T}$. Calculate the derivative $V(t)$ with respect to time $t$ along the trajectory of error system (2.8), and estimate it. 
For $l \omega \leq t \leq l \omega+\delta$, using Lemma 2.3 and assumption, we have the following:

$$
\begin{aligned}
& \dot{V}(t)=\sum_{i=1}^{N} e_{i}^{T}(t) P \dot{e}_{i}(t) \\
& =\sum_{i=1}^{N} e_{i}^{T}(t) P\left[-D e_{i}(t)+A\left(f\left(x_{i}(t)\right)-f(s(t))\right)+B\left(g\left(x_{i}(t-\tau)\right)-g(s(t-\tau))\right)\right. \\
& \left.+C\left(\int_{t-\tau}^{t} h\left(x_{i}(v)\right) d v-\int_{t-\tau}^{t} h(s(v)) d v\right)+\sum_{j=1}^{N} G_{i j} \Gamma e_{j}(t)-k_{i} e_{i}(t)\right] \\
& \leq \sum_{i=1}^{N}\left[-e_{i}^{T}(t) P D e_{i}(t)+\frac{\alpha}{4} e_{i}^{T}(t) P A A^{T} P e_{i}(t)+\alpha^{-1}\left\|f\left(x_{i}(t)\right)-f(s(t))\right\|^{2}\right. \\
& +\frac{\beta}{4} e_{i}^{T}(t) P B B^{T} P e_{i}(t)+\beta^{-1}\left\|g\left(x_{i}(t-\tau)\right)-g(s(t-\tau))\right\|^{2} \\
& \left.+\frac{\gamma}{4} e_{i}^{T}(t) P C C^{T} P e_{i}(t)+\gamma^{-1}\left\|\int_{t-\tau}^{t} h\left(x_{i}(v)\right) d v-\int_{t-\tau}^{t} h(s(v)) d v\right\|^{2}\right] \\
& +\sum_{i=1}^{N} \sum_{j=1}^{N} G_{i j} e_{i}^{T}(t) \Gamma e_{j}(t)-\sum_{i=1}^{N} k_{i} e_{i}^{T}(t) e_{i}(t) \\
& \leq e^{T}(t) I_{N} \otimes\left(-P D+\frac{\alpha}{4} P A A^{T} P+\alpha^{-1} L_{f}^{2} I_{n}\right. \\
& \left.+\frac{\beta}{4} P B B^{T} P+\frac{\gamma}{4} P C C^{T} P+a_{1} P\right) e(t)-a_{1} e^{T}(t)\left(I_{N} \otimes P\right) e(t) \\
& +e^{T}(t-\tau)\left[I_{N} \otimes\left(\beta^{-1} L_{g}^{2} I_{n}-b_{1} P\right)\right] e(t-\tau)+b_{1} e^{T}(t-\tau)\left(I_{N} \otimes P\right) e(t-\tau) \\
& +\left[\int_{t-\tau}^{t} e(v) d v\right]^{T}\left[I_{N} \otimes\left(\gamma^{-1} L_{h}^{2} I_{n}-b_{2} P\right)\right]\left[\int_{t-\tau}^{t} e(v) d v\right] \\
& +b_{2}\left[\int_{t-\tau}^{t} e(v) d v\right]^{T}\left(I_{N} \otimes P\right)\left[\int_{t-\tau}^{t} e(v) d v\right]+e^{T}(t)\left(G \otimes \Gamma-K \otimes I_{n}\right) e(t) .
\end{aligned}
$$

From Jensen's inequality in Lemma 2.4, we have

$$
b_{2}\left[\int_{t-\tau}^{t} e(v) d v\right]^{T}\left(I_{N} \otimes P\right)\left[\int_{t-\tau}^{t} e(v) d v\right] \leq \tau b_{2} \int_{t-\tau}^{t} e^{T}(v)\left(I_{N} \otimes P\right) e(v) d v .
$$

By condition (a), (c), (d), and (3.3), one has

$$
\dot{V}(t) \leq-a_{1} V(t)+b \max V_{t}
$$


For $l \omega+\delta \leq t \leq(l+1) \omega$, from conditions (b), (c), and (d), one has

$$
\dot{V}(t)<a_{2} V(t)+b V_{t}
$$

Next, we will prove the error $\|e(t)\| \rightarrow 0$.

From Lemma 2.5 and (3.2), one has

$$
V(t) \leq\|V(0)\|_{\tau} e^{-r t}, \quad \text { for } 0 \leq t \leq \delta,
$$

where $r$ is the unique positive solution of $-r=-a_{1}+b e^{r \tau}$.

From Lemma 2.6, one obtain the following:

$$
\begin{aligned}
V(t) & \leq\|V(\delta)\|_{\tau} e^{\left(a_{2}+b\right)(t-\delta)} \\
& =\max _{\delta-\tau \leq t \leq \delta}|V(t)| e^{\left(a_{2}+b\right)(t-\delta)} \\
& \leq\|V(0)\|_{\tau} e^{-r(\delta-\tau)} e^{\left(a_{2}+b\right)(t-\delta)},
\end{aligned}
$$

for $\delta \leq t \leq \omega$.

Suppose that $\omega-\tau>\delta$, then

$$
\begin{aligned}
\|V(\omega)\|_{\tau} & =\max _{\omega-\tau \leq t \leq \omega}|V(t)| \\
& \leq \max _{\omega-\tau \leq t \leq \omega}\left\{\|V(0)\|_{\tau} e^{-r(\delta-\tau)} e^{\left(a_{2}+b\right)(t-\delta)}\right\} \\
& =\|V(0)\|_{\tau} e^{-r(\delta-\tau)} e^{\left(a_{2}+b\right)(\omega-\delta)} \\
& =\|V(0)\|_{\tau} e^{-\rho} .
\end{aligned}
$$

Using mathematical induction, we can prove, for any positive integer $l$,

$$
\|V(l \omega)\|_{\tau} \leq\|V(0)\|_{\tau} e^{-l \rho} .
$$

Assume (3.9) holds when $k \leq l$. Now, we prove (3.9) is true when $k=l+1$.

First, we have

$$
\|V(l \omega)\|_{\tau} \leq\|V(0)\|_{\tau} e^{-l \rho} .
$$

When $t \in[l \omega, l \omega+\delta]$,

$$
V(t) \leq\|V(l \omega)\|_{\tau} e^{-r(t-l \omega)} \leq\|V(0)\|_{\tau} e^{-l \rho} e^{-r(t-l \omega)} .
$$


Thus, for $t \in[l \omega+\delta,(l+1) \omega]$, we have

$$
\begin{aligned}
V(t) \leq & \|V(l \omega+\delta)\|_{\tau} e^{\left(a_{2}+b\right)(t-l \omega-\delta)} \\
= & {\left[\max _{l \omega+\delta-\tau \leq t \leq l \omega+\delta} \mid V(t) \|\right] e^{\left(a_{2}+b\right)(t-l \omega-\delta)} } \\
\leq & {\left[\max _{l \omega+\delta-\tau \leq t \leq l \omega+\delta}\|V(0)\|_{\tau} e^{-l \rho} e^{-r(t-l \omega)}\right] } \\
& \times e^{\left(a_{2}+b\right)(t-l \omega-\delta)} \\
\leq & \|V(0)\|_{\tau} e^{-l \rho} e^{-r(\delta-\tau)} e^{\left(a_{2}+b\right)(t-l \omega-\delta)}, \\
\|V((l+1) \omega)\|_{\tau}= & \max _{(l+1) \omega-\tau \leq t \leq(l+1) \omega}|V(t)| \\
\leq & \max _{(l+1) \omega-\tau \leq t \leq(l+1) \omega}\left[\|V(0)\|_{\tau} e^{-l \rho} e^{-r(\delta-\tau)} e^{\left(a_{2}+b\right)(t-l \omega-\delta)}\right] \\
= & \|V(0)\|_{\tau} e^{-l \rho} e^{-r(\delta-\tau)} e^{\left(a_{2}+b\right)(\omega-\delta)} \\
= & \|V(0)\|_{\tau} e^{-(l+1) \rho} .
\end{aligned}
$$

Thus, (3.9) holds for all positive integers $k$.

For any $t>0$, there is $n_{0} \geq 0$, such that $n_{0} \omega \leq t \leq\left(n_{0}+1\right) \omega$;

$$
\begin{aligned}
V(t) & \leq\left\|V\left(n_{0} \omega\right)\right\|_{\tau} e^{\left(a_{2}+b\right)\left(t-n_{0} \omega\right)} \\
& \leq\|V(0)\|_{\tau} e^{-n_{0} \rho} e^{\left(a_{2}+b\right) \omega} \\
& \leq\|V(0)\|_{\tau} e^{\left(a_{2}+b\right) \omega} e^{\rho} \exp \left(-\frac{\rho}{\omega} t\right) .
\end{aligned}
$$

Let $M=\|V(0)\|_{\tau} e^{\left(a_{2}+b\right) \omega} e^{\rho}$, one has the following inequality:

$$
\lambda_{m}(P)\|e(t)\|^{2} \leq V(t) \leq M \exp \left(-\frac{\rho}{\omega} t\right), \quad \text { for } t \geq 0 .
$$

Obviously,

$$
\|e(t)\| \leq \sqrt{\frac{M}{\lambda_{m}(P)}} \exp \left(-\frac{\rho}{2 \omega} t\right)
$$

which means the coupled networks (2.5) achieve synchronization. The proof is completed.

Corollary 3.2. For given control period $\omega$ and control duration $\delta$, coupled networks (2.5) achieve synchronization, if the control gain $K=k I_{N}$ satisfies

$$
k>\Phi\left(r^{*}\right),
$$


where

$$
\begin{gathered}
\Phi(r)=M+r+\left(2 L_{g}^{2}+2 \tau^{2} L_{h}^{2}\right) e^{r \tau}, \\
r^{*}=\frac{\omega-\delta}{\delta-\tau}\left(M+2 L_{g}^{2}+2 \tau^{2} L_{h}^{2}\right) .
\end{gathered}
$$

Proof. In Theorem 3.1, let $P=I, \beta=\gamma=1, b_{1}=2 L_{g}^{2}, b_{2}=2 L_{h^{\prime}}^{2}$ obviously, (c) and (d) in Theorem 3.1 hold.

Furthermore, let $M=\lambda_{M}(Q)+\lambda_{M}(G) \times \lambda_{M}(\Gamma)$, where $\lambda_{M}(\cdot)$ is the maximum of eigenvalue, $a_{1}=k-M>0$ and $a_{2}=M$, (a) and (b) in Theorem 3.1 hold. From the above parameters and (f) in Theorem 3.1 hold if $r>r^{*}$ and $r$ is the positive solution of $-r=-a_{1}+\left(2 L_{g}^{2}+2 \tau^{2} L_{h}^{2}\right) e^{r \tau}$, that is, $k=r+M+L_{f}+\left(2 L_{g}^{2}+2 \tau^{2} L_{h}^{2}\right) e^{r \tau}=\Phi(r)$. Obviously, $\Phi(r)$ is increasing function.

Therefore, (a)-(f) hold if

$$
k>\max \left[\Phi\left(r^{*}\right), M\right]=\Phi\left(r^{*}\right)
$$

Remark 3.3. Corollary 3.2 shows us how to determine the control gain in a simple way provided that the control period $\omega$ and control duration $\delta$ are given.

\section{Numerical Example}

Consider the following coupled networks:

$$
\begin{gathered}
\dot{x}_{i}(t)=-D x_{i}(t)+A f\left(x_{i}(t)\right)+B g\left(x_{i}(t-\tau)\right) \\
+C \int_{t-\tau}^{t} h\left(x_{i}(v)\right) d v+\sum_{j=1}^{N} G_{i j} \Gamma x_{j}(t), \\
x_{i}(t)=\phi(t), \quad-\tau \leq t \leq 0,
\end{gathered}
$$

where $x_{i}(t)=\left[x_{i 1}(t), x_{i 2}(t)\right]^{T}, i=1,2,3$ are the state variable of the $i$ th neural network. Choose $\tau=1, f\left(x_{i}(t)\right)=g\left(x_{i}(t)\right)=h\left(x_{i}(t)\right)=(3 / 5)\left[\tanh \left(x_{i 1}\right), \tanh \left(x_{i 2}\right)\right]^{T}$, and

$$
\begin{array}{ll}
D & =\left[\begin{array}{ll}
1 & 0 \\
0 & 1
\end{array}\right], \quad A=\left[\begin{array}{cc}
2.0 & -0.1 \\
-5.0 & 4.5
\end{array}\right], \\
B & =\left[\begin{array}{cc}
-1.5 & -0.1 \\
-0.2 & -2
\end{array}\right], \quad C=\left[\begin{array}{ll}
-0.2 & -0.1 \\
-1.6 & -3.2
\end{array}\right], \quad G=\left[\begin{array}{ccc}
-4 & 2 & 2 \\
1 & -2 & 1 \\
1 & 2 & -3
\end{array}\right],
\end{array}
$$




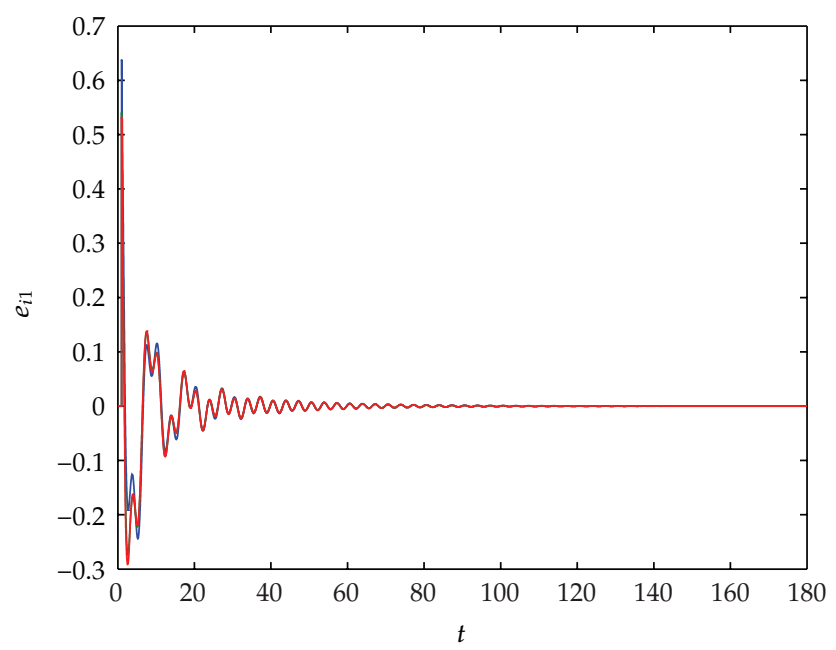

Figure 1: Error state $e_{i 1}(t)$.

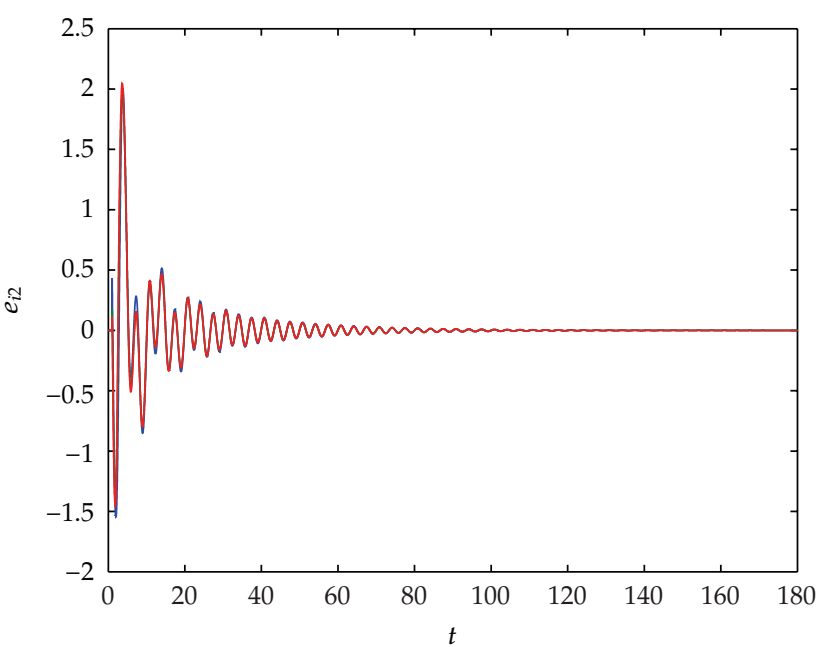

Figure 2: Error state $e_{i 2}(t)$.

and input vectors $I=\left[\begin{array}{l}0 \\ 0\end{array}\right]$, and $k_{i}(t)$ is the intermittent linear state feedback control gain defined as the following:

$$
k_{i}(t)= \begin{cases}k_{i}, & k \omega \leq t \leq k \omega+\delta, \\ 0, & k \omega+\delta<t \leq(k+1) \omega,\end{cases}
$$

where the control gain $k_{1}=k_{2}=k_{3}=0.1$, the control period $\omega=3$, and the control width $\delta=1.3$. The above suitable $\delta, \omega$ and $K$ such that (4.1) synchronize. The synchronize errors are given in Figures 1 and 2. 


\section{Conclusion}

In this paper, synchronization of coupled networks with mixed time delay has been investigated via intermittent control. Some criteria for ensuring coupled networks synchronization have been derived, and some analytical techniques have been proposed to obtain appropriate control period $\omega$, control width $\delta$, and control gain for achieving network synchronization. Finally, the simulation confirmed the effectiveness of the proposed intermittent controller.

\section{Acknowledgments}

This work was jointly supported by the National Natural Science Foundation of China under Grant 61004043 and the Specialized Research Fund for the Doctoral Program of Higher Education under Grant 2009092120066.

\section{References}

[1] D. J. Watts and S. H. Strogatz, “Collective dynamics of 'small-world9 networks," Nature, vol. 393, no. 6684, pp. 440-442, 1998.

[2] A.-L. Barabási and R. Albert, "Emergence of scaling in random networks," Science, vol. 286, no. 5439, pp. 509-512, 1999.

[3] P. Erdős and A. Rényi, “On random graphs. I," Publicationes Mathematicae Debrecen, vol. 6, pp. 290-297, 1959.

[4] P. Erdős and A. Rényi, "On the evolution of random graphs," Publications of the Mathematical Institute of the Hungarian Academy of Sciences, vol. 5, pp. 17-61, 1960.

[5] C. W. Wu and L. O. Chua, "Synchronization in an array of linearly coupled dynamical systems," IEEE Transactions on Circuits and Systems, vol. 42, no. 8, pp. 430-447, 1995.

[6] C. W. Wu, "Synchronization in coupled arrays of chaotic oscillators with nonreciprocal coupling," IEEE Transactions on Circuits and Systems, vol. 50, no. 2, pp. 294-297, 2003.

[7] E. M. Izhikevich and F. C. Hoppensteadt, "Slowly coupled oscillators: phase dynamics and synchronization," SIAM Journal on Applied Mathematics, vol. 63, no. 6, pp. 1935-1953, 2003.

[8] W. Lu and T. Chen, "Synchronization of coupled connected neural networks with delays," IEEE Transactions on Circuits and Systems, vol. 51, no. 12, pp. 2491-2503, 2004.

[9] G. Chen, J. Zhou, and Z. Liu, "Global synchronization of coupled delayed neural networks and applications to chaotic CNN models," International Journal of Bifurcation and Chaos in Applied Sciences and Engineering, vol. 14, no. 7, pp. 2229-2240, 2004.

[10] W. Wang and J. Cao, "Synchronization in an array of linearly coupled networks with time-varying delay," Physica A, vol. 366, pp. 197-211, 2006.

[11] J. Cao, G. Chen, and P. Li, "Global synchronization in an array of delayed neural networks with hybrid coupling," IEEE Transactions on Systems, Man, and Cybernetics B, vol. 38, no. 2, pp. 488-498, 2008.

[12] W. Yu, J. Cao, and J. Lü, "Global synchronization of linearly hybrid coupled networks with timevarying delay," SIAM Journal on Applied Dynamical Systems, vol. 7, no. 1, pp. 108-133, 2008.

[13] W. Yu, J. Cao, G. Chen, J. Lü, J. Han, and W. Wei, "Local synchronization of a complex network model," IEEE Transactions on Systems, Man, and Cybernetics B, vol. 39, no. 1, pp. 230-241, 2009.

[14] K. Yuan, "Robust synchronization in arrays of coupled networks with delay and mixed coupling," Neurocomputing, vol. 72, no. 4-6, pp. 1026-1031, 2009.

[15] J. Cao, P. Li, and W. Wang, "Global synchronization in arrays of delayed neural networks with constant and delayed coupling," Physics Letters A, vol. 353, no. 4, pp. 318-325, 2006.

[16] Z. Gao and S. X. Ding, "State and disturbance estimator for time-delay systems with application to fault estimation and signal compensation," IEEE Transactions on Signal Processing, vol. 55, no. 12, pp. 5541-5551, 2007.

[17] Z. Gao, T. Breikin, and H. Wang, "Reliable observer-based control against sensor failures for systems with time delays in both state and input," IEEE Transactions on Systems, Man, and Cybernetics A, vol. 38, no. 5, pp. 1018-1029, 2008.

[18] M. Zochowski, “Intermittent dynamical control,” Physica D, vol. 145, no. 3-4, pp. 181-190, 2000. 
[19] C. Li, X. Liao, and T. Huang, "Exponential stabilization of chaotic systems with delay by periodically intermittent control," Chaos, vol. 17, no. 1, article 013103, 2007.

[20] C. Li, G. Feng, and X. Liao, "Stabilization of nonlinear systems via periodically intermittent control," IEEE Transactions on Circuits and Systems II, vol. 54, no. 11, pp. 1019-1023, 2007.

[21] T. Huang, C. Li, and X. Liu, "Synchronization of chaotic systems with delay using intermittent linear state feedback," Chaos, vol. 18, no. 3, article 033122, 2008.

[22] T. Huang, C. Li, W. Yu, and G. Chen, "Synchronization of delayed chaotic systems with parameter mismatches by using intermittent linear state feedback," Nonlinearity, vol. 22, no. 3, pp. 569-584, 2009.

[23] X. Yang and J. Cao, "Stochastic synchronization of coupled neural networks with intermittent control," Physics Letters A, vol. 373, no. 36, pp. 3259-3272, 2009.

[24] S. Cai, Z. Liu, F. Xu, and J. Shen, "Periodically intermittent controlling complex dynamical networks with time-varying delays to a desired orbit," Physics Letters A, vol. 373, no. 42, pp. 3846-3854, 2009.

[25] W. Xia and J. Cao, "Pinning synchronization of delayed dynamical networks via periodically intermittent control," Chaos, vol. 19, no. 1, article 013120, 2009.

[26] X. Liu and T. Chen, "Cluster synchronization in directed networks via intermittent pinning control," IEEE Transactions on Neural Networks, vol. 22, no. 7, pp. 1009-1020, 2011.

[27] J. C. Principe, J. M. Kuo, and S. Celebi, "Analysis of the gamma memory in dynamic neural networks," IEEE Transactions on Neural Networks, vol. 5, no. 2, pp. 331-337, 1994.

[28] Z. Wang, Y. Liu, and X. Liu, "On global asymptotic stability of neural networks with discrete and distributed delays," Physics Letters A, vol. 345, no. 4-6, pp. 299-308, 2005.

[29] S. Ruan and R. S. Filfil, "Dynamics of a two-neuron system with discrete and distributed delays," Physica D, vol. 191, no. 3-4, pp. 323-342, 2004.

[30] J. Cao, K. Yuan, D. W. C. Ho, and J. Lam, “Global point dissipativity of neural networks with mixed time-varying delays," Chaos, vol. 16, no. 1, article 013105, 2006.

[31] Z. Wang, Y. Liu, M. Li, and X. Liu, "Stability analysis for stochastic Cohen-Grossberg neural networks with mixed time delays," IEEE Transactions on Neural Networks, vol. 17, no. 3, pp. 814-820, 2006.

[32] Y. Liu, Z. Wang, and X. Liu, "Global exponential stability of generalized recurrent neural networks with discrete and distributed delays," Neural Networks, vol. 19, no. 5, pp. 667-675, 2006.

[33] K. Yuan, J. Cao, and H. X. Li, "Robust stability of switched Cohen-Grossberg neural networks with mixed time-varying delays," IEEE Transactions on Systems, Man, and Cybernetics B, vol. 36, no. 6, pp. 1356-1363, 2006.

[34] K. Gu, V. Kharitonov, and J. Chen, Stability of Time-Delay Systems, Birkhauser, Boston, Mass, USA, 2003.

[35] A. Halanay, Differential Equations: Stability, Oscillations, Time Lags, Academic Press, New York, NY, USA, 1966. 


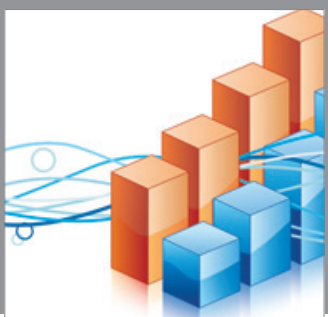

Advances in

Operations Research

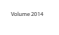

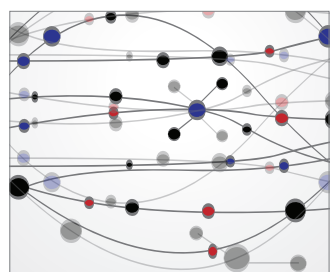

\section{The Scientific} World Journal
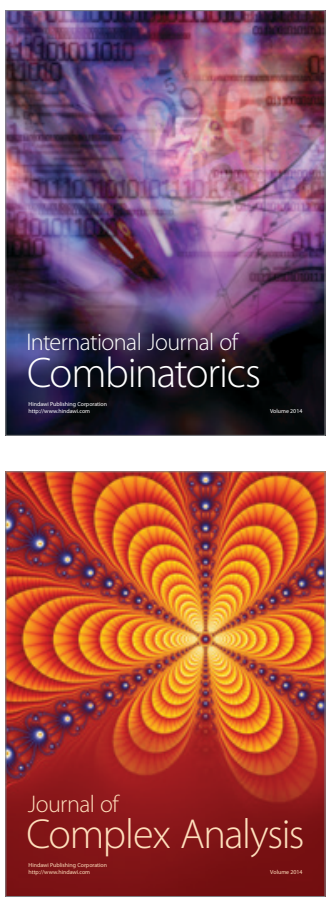

International Journal of

Mathematics and

Mathematical

Sciences
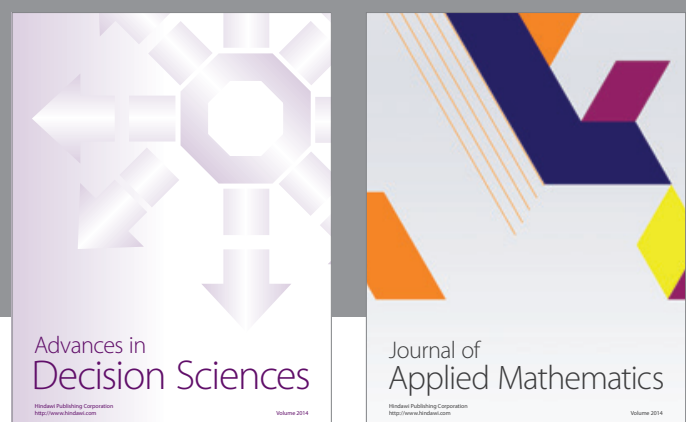

Journal of

Applied Mathematics
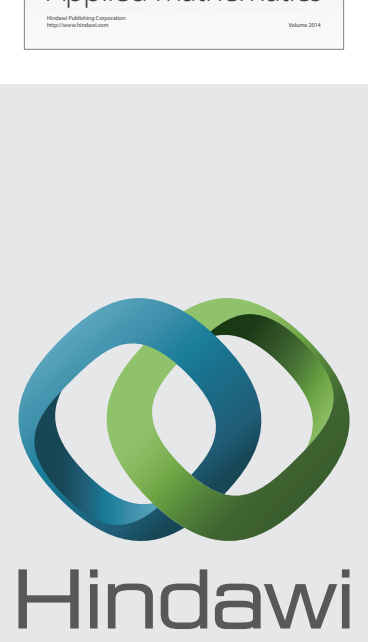

Submit your manuscripts at http://www.hindawi.com
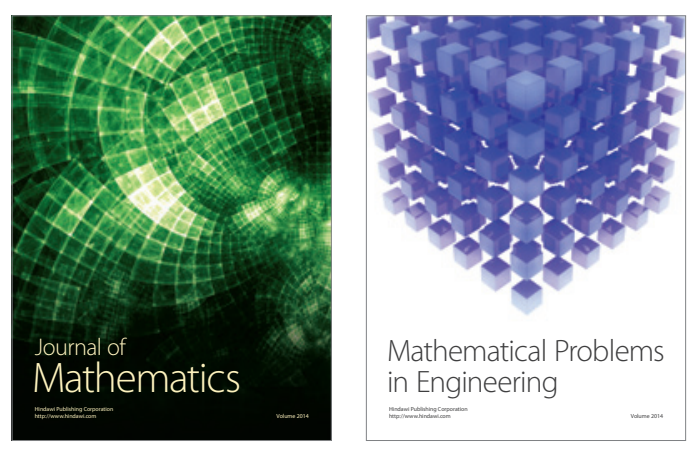

Mathematical Problems in Engineering
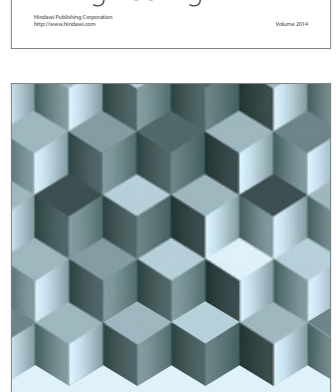

Journal of

Function Spaces
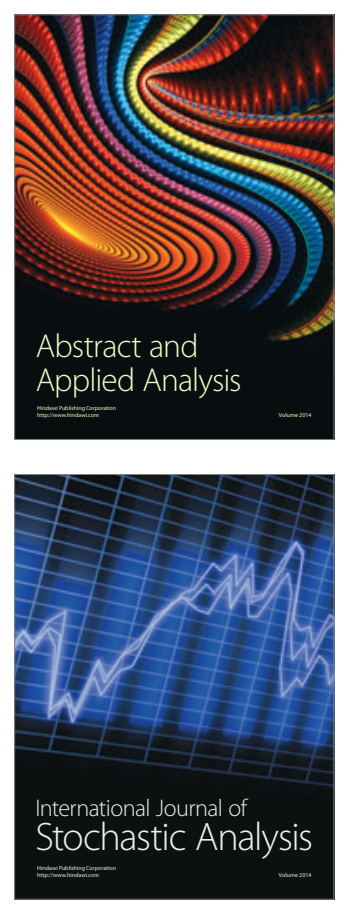

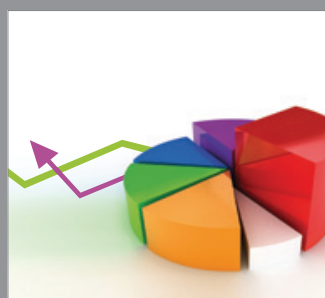

ournal of

Probability and Statistics

Promensencen
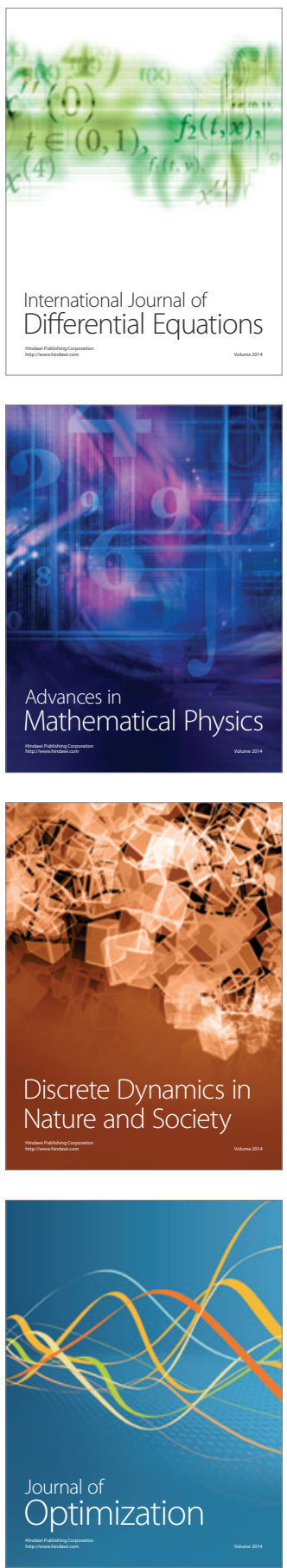